\title{
DIVISIBILITY CONSTRAINTS ON DEGREES OF FACTOR MAPS
}

\author{
PAUL TROW
}

(Communicated by Kenneth R. Meyer)

\begin{abstract}
We show that the degree of a finite-to-one factor map $f: \sum_{A} \rightarrow$ $\sum_{B}$ between shifts of finite type is constrained by the factors of $\chi_{A}$ and $\chi_{B}$. A special case of these constraints is that if $2 \operatorname{rank}^{*} B>\operatorname{rank}^{*} A$, then the degree of $f$ is a unit in $\mathbb{Z}\left[1 / \operatorname{det}^{*} B\right]$ (where $\operatorname{rank}^{*} A$ is the rank of the Jordan form away from 0 of $A$, and $\operatorname{det}^{*} B$ is the determinant of the Jordan form away from 0 of $B$ ).
\end{abstract}

\section{INTRODUCTION}

The degree of a finite-to-one factor map $f: \sum_{A} \rightarrow \sum_{B}$ between irreducible shifts of finite type is the largest positive integer $d$ such that every $y \in \sum_{B}$ has $d$ mutually separated inverse images. This concept was introduced in [H] for endomorphisms of full shifts. In [B], M. Boyle showed that, given $\sum_{A}$ and $\sum_{B}$, there exists a finite set $E$ of positive integers such that if $f: \sum_{A} \rightarrow \sum_{B}$ has degree $d$, then $d=e u$, where $e \in E$ and $u$ is a unit in $\mathbb{Z}[1 / \lambda]$, where $\lambda$ is the dominant eigenvalue of $A$. In [T2], this result was extended to apply to any nonzero eigenvalue $\gamma$ that has the same algebraic multiplicity in $\chi_{A}$ and $\chi_{B}$. In this paper we give further constraints on the possible degrees of a finiteto-one factor map, given certain assumptions on the factors of $\chi_{A}$ and $\chi_{B}$ (see Theorem 8 and Corollary 9).

\section{BACKGROUND}

We give some basic definitions concerning shifts of finite type. For a more thorough introduction to symbolic dynamics, see [AM] or [PT].

Let $A$ be an $n \times n$, nonnegative, integral matrix, and let $G(A)$ be the directed graph on $n$ vertices, with $A_{i j}$ edges from vertex $i$ to vertex $j$. Let $\mathscr{E}_{A}=$ \{edges of $G(A)\}$. We define the shift of finite type $\sum_{A}=\left\{x \in \mathscr{E}_{A}^{\mathbb{Z}}: x_{i+1}\right.$ follows $x_{i}$ for all $i$, where $x_{i+1}$ follows $x_{i}$ if the terminal vertex of $x_{i}$ is the initial vertex of $x_{i+1} \cdot \sum_{A}$ is a compact metric space under the product topology.

$A$ is irreducible if for each $i, j$, there exists $n=n(i, j)$ such that $A_{i j}^{n}>$ 0 . $\sum_{A}$ is irreducible if $A$ is. A factor map $f: \sum_{A} \rightarrow \sum_{B}$ is a continuous,

Received by the editors April 28, 1990.

1980 Mathematics Subject Classification (1985 Revision). Primary 28D05. 
surjective map which commutes with the shift. $f$ is finite-to-one if there exists $N \in \mathbb{Z}^{+}$such that $\# f^{-1}(y) \leq N$, for all $y \in \sum_{B}$. By [CP, Corollary 4.5], this is equivalent to $h\left(\sum_{A}\right)=h\left(\sum_{B}\right)$.

If $D$ is a principal ideal domain, and $g, h \in D$, we denote the greatest common divisor of $g$ and $h$ by $(g, h)$. If $p(x) \in \mathbb{Z}[x]$, we denote the degree of $p(x)$ by $\operatorname{deg} p(x)$.

If $A$ is a square, integral matrix, and $\chi_{A}=p(x) x^{r}$, where $r$ is the largest power of $x$ which divides $\chi_{A}$, let $\chi_{A}^{*}=p(x), \operatorname{rank}^{*} A=\operatorname{deg} p(x)$, and $\operatorname{det}^{*} A=$ constant term of $p(x)$.

If $F$ is a field, $A$ is a square matrix over $F$, and $g(x)$ is a factor of $\chi_{A}$, we denote the null space of $g(A)$ by $N(g(A))$. If $V$ is a vector space over $F$, we denote the dual space of $V$ by $V^{*}$. If $W$ is a subspace of $V$, the annihilator of $W$, denoted Ann $W$, is $\left\{f \in V^{*}: f(w)=0\right.$ for all $\left.w \in W\right\}$.

If $\mathscr{L} \subseteq \mathbb{Z}^{n}, \mathscr{L}$ is called a sublattice. $\mathscr{L}$ is closed if $\mathscr{L}=\langle\mathscr{L}\rangle \cap \mathbb{Z}^{n}$, where $\langle\mathscr{L}\rangle$ denotes the rational span of $\mathscr{L}$.

\section{MAIN RESUlts}

Definition 1. Let $p(x)$ be a monic, integral polynomial, and $p(x)=\prod_{i=1}^{t} p_{i}^{e_{i}}(x)$ be the unique factorization of $p(x)$ in $\mathbb{Z}[x]$, where the $p_{i}(x)$ are distinct, monic, irreducible polynomials. Any factor of $p(x)$ which is a product of some of the $p_{i}^{e_{i}}(x)$ is called a complete factor of $p(x)$. If $g(x)$ is a complete factor of $p(x)$, and $h(x)$ is a complete factor of $p(x) / g(x)$, then $h(x)$ is called a complementary complete factor of $p(x)$.

If $f: \sum_{A} \rightarrow \sum_{B}$ is a finite-to-one factor map, then $\chi_{B}^{*}$ divides $\chi_{A}^{*}$ (see [K, Corollary A]). If $g_{B}(x)=\prod p_{i}^{e_{i}}(x)$ is a complete factor of $p(x)=\chi_{B}^{*}$, then $g_{A}(x)=\prod p_{i}^{f_{i}}(x), f_{i} \geq e_{i}$, is a complete factor of $\chi_{A}^{*}$, where $f_{i}$ is the power to which $p_{i}$ is raised in $\chi_{A}^{*}$. Then $g_{A}(x)$ is called the corresponding complete factor of $\chi_{A}^{*}$.

Lemma 2. Let $B$ be a $k \times k$ integral matrix, and let $g(x)$ be a complete factor of $\chi_{B}$. Then there is a closed, B-invariant sublattice $\mathscr{L} \subseteq N(g(B))$ such that rank $\mathscr{L}=\operatorname{deg} g(x)$.

Proof. Let $\mathscr{L}=N(g(B)) \cap \mathbb{Z}^{k}$. By the Primary Decomposition Theorem ([HK, Theorem 12, p. 189]), rank $\mathscr{L}=\operatorname{deg} g(x)$.

We now consider a sublattice $\mathscr{L}$ reduced over $\mathbb{Z} / p \mathbb{Z}$, for $p$ a prime. Lemmas 4-6 are elementary results from linear algebra.

Definition 3. For $p$ a fixed prime, let $\pi: \mathbb{Z} \rightarrow \mathbb{Z} / p \mathbb{Z}$ denote the natural homomorphism $k \rightarrow k+p \mathbb{Z}$. Denote $\pi(k)$ by $\bar{k}$. If $A=\left(a_{i j}\right)$ is an $n \times n$ integral matrix, let $\bar{A}$ denote the $n \times n$ matrix over $\mathbb{Z} / p \mathbb{Z}$ given by $\bar{A}_{i j}=\bar{a}_{i j}$. If $v=\left(v_{1}, \ldots, v_{n}\right) \in \mathbb{Z}^{n}$, let $\bar{v} \in(\mathbb{Z} / p \mathbb{Z})^{n}$ be defined by $\bar{v}=\left(\bar{v}_{1}, \ldots, \bar{v}_{n}\right)$. If $\mathscr{L} \subseteq \mathbb{Z}^{n}$, let $\overline{\mathscr{L}}=\{\bar{v}: v \in \mathscr{L}\}$. 
Lemma 4. If $A$ is an $n \times n$ integral matrix and $\mathscr{L} \subseteq \mathbb{Z}^{n}$ is a closed sublattice, then $\overline{\mathscr{L}}$ is an $\bar{A}$-invariant subspace of $(\mathbb{Z} / p \mathbb{Z})^{n}$ and $\operatorname{rank} \mathscr{L}=\operatorname{dim} \overline{\mathscr{L}}$.

Proof. It is easy to check that $\mathscr{L}$ is an $\bar{A}$-invariant subspace. To see that rank $\mathscr{L}=\operatorname{dim} \overline{\mathscr{L}}$, choose an integral basis $\mathscr{B}=\left\{\beta_{1}, \ldots, \beta_{k}\right\}$ for $\mathscr{L}$. We claim that $\overline{\mathscr{B}}=\left\{\bar{\beta}_{1}, \ldots, \bar{\beta}_{k}\right\}$ is a basis for $\overline{\mathscr{L}}$. If $\bar{v} \in \overline{\mathscr{L}}$, where $v \in \mathscr{L}$, then $v \in \sum_{i=1}^{k} c_{i} \beta_{i}$, for some $c_{i} \in \mathbb{Z}$, since $\mathscr{B}$ is an integral basis for $\mathscr{L}$. Therefore $\bar{v}=\sum_{i=1}^{k} \bar{c}_{i} \bar{\beta}_{i}$, so $\mathscr{B}$ spans $\overline{\mathscr{L}}$. If $\sum_{i=1}^{k} \bar{c}_{i} \bar{\beta}_{i}=(\overline{0}, \ldots, \overline{0})$, then $p$ divides $\left(\sum_{i=1}^{k} c_{i} \beta_{i}\right)_{j}$, for $1 \leq j \leq n$. It follows that $v=\sum_{i=1}^{k}\left(c_{i} / p\right) \beta_{i} \in \mathscr{L}$, since $v \in\langle\mathscr{L}\rangle \cap \mathbb{Z}^{n}$ and $\mathscr{L}$ is closed. This implies that $c_{i} / p \in \mathbb{Z}$, since $\mathscr{B}$ is an integral basis for $\mathscr{L}$. Therefore $\bar{c}_{i}=\overline{0}$, so $\mathscr{B}$ is linearly independent. It follows that $\operatorname{rank} \mathscr{L}=\operatorname{dim} \overline{\mathscr{L}}$.

Lemma 5. Let $\bar{A}$ be an $n \times n$ matrix over a field $F$. Let $\bar{g}(x)$ and $\bar{h}(x)$ be factors of $\chi_{\bar{A}}$. If $(\bar{g}(x), \bar{h}(x))=1$ in $F[x]$, and $v \in N(\bar{h}(\bar{A})), w \in N\left(\bar{g}\left(\bar{A}^{T}\right)\right)$, then $v w^{T}=0$.

Proof. Because $(\bar{g}(x), \bar{h}(x))=1$, there exist $q(x), r(x) \in F[x]$ such that $\bar{g}(x) q(x)+\bar{h}(x) r(x)=1$. Therefore $v=v \bar{g}(A) q(A)$ and $w^{T}=\bar{h}(A) r(A) w^{T}$. So $v w^{T}=v \bar{g}(A) q(A) \bar{h}(A) r(A) w^{T}=v \bar{h}(A) q(A) r(A) \bar{g}(A) w^{T}=0$.

Lemma 6. Let $U$ and $V$ be subspaces of $(\mathbb{Z} / p \mathbb{Z})^{n}$. Assume that $\bar{v} \bar{w}^{T}=\overline{0}$ for all $\bar{v} \in U, \bar{w} \in V$. Then $\operatorname{dim} U \leq \operatorname{dim} \operatorname{Ann}(V)$.

Proof. It is easy to check that the linear map $\phi:(\mathbb{Z} / p \mathbb{Z})^{n} \rightarrow(\mathbb{Z} / p \mathbb{Z})^{n^{*}}$ given by $v \rightarrow f_{v}$, where $f_{v}(w)=v w^{T}$, is injective. Since $\phi(U) \subseteq \operatorname{Ann}(V)$, the result follows.

Theorem 7. Let $A$ be an $n \times n$ integral matrix, $p$ a prime, $g(x)$ a complete factor of $\chi_{A}^{*}$, with constant term $c$, and $h(x)$ a complementary complete factor of $\chi_{A}^{*}$. Assume that $(\bar{g}(x), \bar{h}(x))=\overline{1}$ and that $(p, c)=1$. Let $\mathscr{L}_{1}$ be a closed, $A$-invariant sublattice of $N(g(A))$ and $\mathscr{L}_{2}$ a closed, $A^{T}$-invariant sublattice of $N\left(g\left(A^{T}\right)\right)$. If $p$ divides $v w^{T}$, for all $v \in \mathscr{L}_{1}, w \in \mathscr{L}_{2}$, then rank $\mathscr{L}_{1}+$ $\operatorname{rank} \mathscr{L}_{2} \leq \operatorname{rank}^{*} A-\operatorname{deg} h(x)$.

Proof. Let $\operatorname{deg} h(x)=s$, and let $r$ be the largest power of $x$ which divides $\chi_{A}$. Clearly, $\operatorname{rank}^{*} A=n-r$. Since $(p, c)=1$, it is easy to see that $(\bar{g}(x), \bar{x})=\overline{1}$. Let $h_{1}(x)=h(x) x^{r}$. Then $\left(\bar{g}(x), \bar{h}_{1}(x)\right)=\overline{1}$. We have $\operatorname{dim} N\left(h_{1}(A)\right) \leq \operatorname{dim} N\left(\bar{h}_{1}(\bar{A})\right)$, since $N\left(h_{1}(A)\right) \cap \mathbb{Z}^{n}$ projects onto a subspace of $N\left(\bar{h}_{1}(\bar{A})\right)$ whose dimension equals rank $N\left(h_{1}(A)\right) \cap \mathbb{Z}^{n}$, by Lemma 4. Also, $\operatorname{dim} N\left(h_{1}(A)\right)=\operatorname{deg} h_{1}(x)=s+r$ by the Primary Decomposition Theorem ([HK, Theorem 12]), since $h_{1}(x)$ is a complete factor of $\chi_{A}$. So $\operatorname{dim} N\left(\bar{h}_{1}(\bar{A})\right) \geq s+r$. Also, we have $\bar{v} \bar{w}^{T}=\overline{0}$, for all $\bar{v} \in \overline{\mathscr{L}}_{1}, \bar{w} \in \overline{\mathscr{L}}_{2}$, since $p$ divides $v w^{T}$. Let $U$ be the span over $\mathbb{Z} / p \mathbb{Z}$ of $N\left(\bar{h}_{1}(\bar{A})\right)$ and $\overline{\mathscr{L}}_{1}$. Since $\overline{\mathscr{L}}_{1} \subseteq N(\bar{g}(\bar{A}))$ and $\left(\bar{g}(x), \bar{h}_{1}(x)\right)=\overline{1}$, it is easy to see that $N\left(\bar{h}_{1}(\bar{A})\right) \cap \overline{\mathscr{L}}_{1}=$ $\{0\}$, so that $\operatorname{dim} U=\operatorname{dim} N\left(\bar{h}_{1}(\bar{A})\right)+\operatorname{dim} \overline{\mathscr{L}}_{1} \geq s+r+\operatorname{dim} \overline{\mathscr{L}}_{1}$. Also, since 
$\overline{\mathscr{L}}_{2} \subseteq N\left(\bar{g}\left(\bar{A}^{T}\right)\right)$, it follows from Lemma 5 that $\bar{v} \bar{w}^{T}=\overline{0}$, for all $\bar{v} \in U$, $\bar{w} \in \overline{\mathscr{L}}_{2}$. By Lemma $6, s+r+\operatorname{dim} \overline{\mathscr{L}}_{1} \leq \operatorname{dim} U \leq \operatorname{dim} \operatorname{Ann}\left(\overline{\mathscr{L}}_{2}\right)$. Since $\operatorname{dim} \overline{\mathscr{L}}_{2}+\operatorname{dim} \operatorname{Ann}\left(\overline{\mathscr{L}}_{2}\right)=\operatorname{dim}(\mathbb{Z} / p \mathbb{Z})^{n}=n$ (by [HK, Theorem 17, p. 98]), we have $s+r+\operatorname{dim} \overline{\mathscr{L}}_{1}+\operatorname{dim} \overline{\mathscr{L}}_{2} \leq n$ or $\operatorname{dim} \overline{\mathscr{L}}_{1}+\operatorname{dim} \overline{\mathscr{L}}_{2} \leq n-r-s=$ $\operatorname{rank}^{*} A-\operatorname{deg} h(x)$. Since $\operatorname{dim} \overline{\mathscr{L}}_{1}=\operatorname{rank} \mathscr{L}_{1}$ and $\operatorname{dim} \overline{\mathscr{L}}_{2}=\operatorname{rank} \mathscr{L}_{2}$, by Lemma 4 , the result follows.

Theorem 8. Suppose that $\sum_{A}$ and $\sum_{B}$ are irreducible shifts of finite type, and that $f: \sum_{A} \rightarrow \sum_{B}$ is a finite-to-one factor map of degree $d$. Suppose that $g_{B}(x)$ is a complete factor of $\chi_{B}^{*}$ and $g_{A}(x)$ is the corresponding complete factor of $\chi_{A}^{*}$, where the constant term of $g_{A}(x)$ is $c$, and that $h(x)$ is a complementary complete factor of $\chi_{A}^{*}$. Let $p$ be a prime which divides $d$, and assume that $\left(\bar{g}_{A}(x), \bar{h}(x)\right)=\overline{1}$ and that $2 \operatorname{deg} g_{B}(x)>\operatorname{rank}^{*} A-\operatorname{deg} h(x)$. Then $p$ divides $c$.

Proof. By [KMT, Theorem 2.3] and [T2, Definition 1.4 and Lemma 1.6] (substituting $\mathbb{Q}$ for $\mathbb{C}$ ), there exist $\sum_{\tilde{A}}$ topologically conjugate to $\sum_{A}$, and $\sum_{\tilde{B}}$ conjugate to $\sum_{B}$, a rational $\tilde{A}$-invariant subspace $V$, a rational $\tilde{A}^{T}$-invariant subspace $W$, and surjective linear maps $\phi: V \rightarrow \mathbb{Q}^{k}$ and $\theta: W \rightarrow \mathbb{Q}^{k}$ (where $\tilde{B}$ is $k \times k)$ such that $v \tilde{A} \phi=v \phi \tilde{B}$ and $w \tilde{A}^{T} \theta=w \theta \tilde{B}^{T}$, for all $v \in V, w \in W$. By [W, Corollary 4.8], $\chi_{A}^{*}=\chi_{\tilde{A}}^{*}$ and $\chi_{B}^{*}=\chi_{\tilde{B}}^{*}$, so $g_{B}(x)$ is a complete factor of $\chi_{\tilde{B}}^{*}$, $g_{A}(x)$ is the corresponding complete factor of $\chi_{\tilde{A}}^{*}$, and $h(x)$ is a complementary complete factor of $\chi_{\tilde{A}}^{*}$. It follows from Lemma 2 and duality that there exist a closed $\tilde{A}$-invariant sublattice $\tilde{\mathscr{L}}_{1} \subseteq N\left(g_{A}(\tilde{A})\right) \cap V$ and a closed $\tilde{A}^{T}$-invariant sublattice $\tilde{\mathscr{L}}_{2} \subseteq N\left(g_{A}\left(\tilde{A}^{T}\right) \cap W\right.$ such that $\operatorname{rank} \tilde{\mathscr{L}}_{1}=\operatorname{rank} \tilde{\mathscr{L}}_{2}=\operatorname{deg} g_{B}(x)$. So $\operatorname{rank} \tilde{\mathscr{L}}_{1}+\operatorname{rank} \tilde{\mathscr{L}}_{2}=2 \operatorname{deg} g_{B}(x)>\operatorname{rank}^{*} A-\operatorname{deg} h(x)$. In [T2, Theorem 2.5], it is shown that if $v \in V$ and $w \in W$ are $\mathbb{Z}$-vectors, then $d$ divides $v w^{T}$, and so $p$ divides $v w^{T}$. If $(p, c)=1$, then by Theorem 7 there would exist $v \in \tilde{\mathscr{L}}_{1}$ and $w \in \tilde{\mathscr{L}}_{2}$ such that $p$ does not divide $v w^{T}$, contradicting the previous statement. Therefore $p$ divides $c$.

Corollary 9. Suppose that $\sum_{A}$ and $\sum_{B}$ are irreducible shifts of finite type, and $f: \sum_{A} \rightarrow \sum_{B}$ is a finite-to-one factor map of degree $d$. If $2 \mathrm{rank}^{*} B>\operatorname{rank}^{*} A$, then every prime which divides $d$ also divides $\operatorname{det}^{*} B$. Therefore $d$ is a unit in $\mathbb{Z}\left[1 / \operatorname{det}^{*} B\right]$.

Proof. Take $g_{B}(x)=\chi_{B}^{*}, g_{A}(x)=\chi_{A}^{*}$, and $h(x)=1$. For any prime $p$, the condition $\left(\bar{g}_{B}(x), \bar{h}(x)\right)=\overline{1}$ holds trivially. Since $\operatorname{deg} g_{B}(x)=\operatorname{rank}^{*} B$, $\operatorname{deg} g_{A}(x)=\operatorname{rank}^{*} B$, and the constant term of $\chi_{B}^{*}$ is $\operatorname{det}^{*} B$, the result follows from Theorem 8 .

Example 10. Suppose that $\sum_{A}$ and $\sum_{B}$ are irreducible shifts of finite type, and that $\chi_{A}^{*}=(x-2)(x-1)^{3}$ and $\chi_{B}^{*}=(x-2)(x-1)^{2}$. Take $g_{B}(x)=$ $(x-1)^{2}, g_{A}(x)=(x-1)^{3}$, and $h(x)=x-2$. Observe that for any prime $p$, $\left(\bar{g}_{A}(x), \bar{h}(x)\right)=\overline{1}$. Since $2 \operatorname{deg} g_{B}(x)=4>\operatorname{rank}^{*} A-\operatorname{deg} h(x)=4-1=3$, 
and the constant term of $g_{A}(x)$ is 1 , if $f: \sum_{A} \rightarrow \sum_{B}$ is a factor map, then the degree of $f$ is 1 , by Theorem 8 .

We remark that if we assume only that $\chi_{A}^{*}=(x-2)(x-1)^{2}$ and $\chi_{B}^{*}=$ $(x-2)(x-1)$, we cannot draw the same conclusion. However, by applying Corollary 9 , we can conclude that the degree of $f$ is a power of 2 , but we do not have an example of a factor map between shifts with the given characteristic polynomials (modulo $x$ ) which has degree 2. Does such a factor map exist?

Example 11. This example shows that Corollary 9 is sharp, in general. Let

$$
A=\left[\begin{array}{llll}
1 & 0 & 0 & 1 \\
0 & 1 & 1 & 0 \\
1 & 0 & 0 & 0 \\
0 & 1 & 0 & 0
\end{array}\right] \text { and } B=\left[\begin{array}{ll}
1 & 1 \\
1 & 0
\end{array}\right]
$$

Then there exists a factor map $f: \sum_{A} \rightarrow \sum_{B}$, of degree 2 , which is right and left resolving (and therefore constant-to-one). Then $f$ is defined as a map on states, sending states 1 and 2 of $\sum_{A}$ to state 1 of $\sum_{B}$ and states 3 and 4 of $\sum_{A}$ to state 2 of $\sum_{A}$. Note that $\operatorname{det}^{*} B=-1$, so 2 does not divide $\operatorname{det}^{*} B$, but that $2 \operatorname{rank}^{*} B=4=\operatorname{rank}^{*} A$.

Example 12. This example shows that the condition that $(\bar{g}(x), \bar{h}(x))=\overline{1}$ in Theorem 8 is necessary. Let

$$
A=\left[\begin{array}{llll}
1 & 0 & 0 & 2 \\
0 & 1 & 2 & 0 \\
1 & 0 & 0 & 0 \\
0 & 1 & 0 & 0
\end{array}\right] \text { and } B=\left[\begin{array}{ll}
1 & 2 \\
1 & 0
\end{array}\right]
$$

There exists a factor map $f: \sum_{A} \rightarrow \sum_{B}$ of degree 2 , similar to the one in the previous example, and $f$ maps states 1 and 2 of $\sum_{A}$ to state 1 of $\sum_{B}$ and states 3 and 4 of $\sum_{A}$ to state 2 of $\sum_{B}$. Let $p=2$. Observe that $\chi_{B}=(x-2)(x+1)$ and $\chi_{A}=(x-2)(x+1)\left(x^{2}-x+2\right)$. Let $g_{B}(x)=x+1=g_{A}(x)$ and $h(x)=$ $(x-2)\left(x^{2}-x+2\right)$. Note that $2 \operatorname{deg} g_{B}(x)=2>\operatorname{rank}^{*} A-\operatorname{deg} h(x)=4-3=1$, but that $\left(\bar{g}_{A}(x), \bar{h}(x)\right) \neq \overline{1}$, since $\bar{g}_{A}(x)=x+1$ and $\bar{h}(x)=x^{2}(x+1)$. Also, the conclusion of Theorem 8 does not hold, since the degree of $f$ is not 1 .

\section{REFERENCES}

[AM] R. L. Adler and B. Marcus, Topological entropy and equivalence of dynamical systems, Mem. Amer. Math. Soc., no. 219, Amer. Math. Soc., Providence, RI, 1979.

[B] M. Boyle, Constraints on the degree of a sofic homomorphism and the induced multiplication of measures on unstable sets, Israel J. Math. 53 (1986), 52-68.

[CP] E. Coven and M. Paul, Sofic systems, Israel J. Math. 20 (1975), 165-177.

[H] G. A. Hedlund, Endomorphisms and automorphisms of the shift dynamical system, Math. Systems Theory 3 (1969), 320-375.

[HK] K. Hoffman and R. Kunze, Linear algebra, Prentice-Hall, Englewood Cliffs, NJ, 1961.

[K] B. Kitchens, An invariant for continuous factors of Markov chains, Proc. Amer. Math. Soc. 83 (1981), 825-828. 
[KMT] B. Kitchens, B. Marcus, and P. Trow, Eventual factor maps and compositions of closing maps, Ergodic Theory Dynamical Systems (to appear).

[PT] W. Parry and S. Tuncel, Classification problems in ergodic theory, London Math. Soc. Lecture Notes Ser., vol. 67, Cambridge University Press, Cambridge and New York, 1982.

[T1] P. Trow, Degrees of constant-to-one factor maps, Proc. Amer. Math. Soc. 103 (1988), 184188.

[T2] _ Degrees of finite-to-one factor maps, Israel J. Math. (to appear).

[W] R. F. Williams, Classification of one-dimensional attractors, Proc. Sympos. Pure Math., vol. 14, Amer. Math. Soc., Providence, RI, 1970, 341-361.

Department of Mathematical Sciences, Memphis State University, Memphis, TennesSEE 38152 